\title{
A COVID-19 Acute Gastroenteritis with Similarity Pneumonia Management; Dramatic Response
}

\author{
Yasser Mohammed Hassanain Elsayed* \\ Critical Care Unit, Fraskour Central Hospital, Damietta Health Affairs, Egyptian Ministry of Health \\ (MOH), Damietta, Egypt \\ *Corresponding Author: Yasser Mohammed Hassanain Elsayed, Critical Care Unit, Fraskour \\ Central Hospital, Damietta Health Affairs, Egyptian Ministry of Health (MOH), Damietta, Egypt
}

\begin{abstract}
Rationale: Coronavirus disease is a wide-spread communicable serious pandemic worldwide infection. COVID-19 infection with acute gastroenteritis is an uncommon possible primary presentation. COVID-19 pneumonia is usually managed with oxygenation, anti-infective drugs, anticoagulants, antiplatelet, steroids, and intravenous fluids.

Patient concerns: An elderly female COVID-19 patient presented to the emergency department with acute gastroenteritis.

Diagnosis: COVID-19 causing acute gastroenteritis.

Interventions: Chest CT scan and intravenous fluids.

Outcomes: Dramatic clinical improvement had happened.

Lessons: It denotes the role of the anti-infective drugs, anticoagulants, antiplatelet, and steroids, intravenous fluids in COVID-19 gastroenteritis patients are effective therapies. With the avoidance of oxygen inhalation, there are closed similarities between COVID-19 pneumonia and COVID-19 gastroenteritis management.
\end{abstract}

Keywords: COVID-19, Coronavirus, Acute gastroenteritis, Pneumonia, Similarity pneumonia management

Abbreviations: CBC: Complete blood count : COVID-19: Coronavirus disease 2019: IV: Intravenous: SARS-CoV-2: Severe acute respiratory syndrome coronavirus 2 : SGOT: Serum glutamic-oxaloacetic transaminase: SGPT: Serum glutamic-pyruvic transaminase : VR: Ventricular rate

\section{INTRODUCTION}

Severe acute respiratory syndrome coronavirus 2 (SARS-CoV-2) or 2019-nCoV emerged in the boycott of Wuhan, China in Dec 2019. SARSCoV-2 was the source of severe respiratory disease coronavirus disease 2019 (COVID-19) [1]. On March 11, 2020, the World Health Organization (WHO) declared that the viral revolution of a novel COVID-19 had spread to a pandemic level [2]. The outcome of the coronavirus COVID-19 is a subversive and considerable public health issue worldwide. SARS-CoV-2 infection related to gastrointestinal alterations. It may be linked to particular phases of COVID-19 correlating to inflammation. The early viral phase is usually marked by an elevated viral load, and a decreased inflammatory activity is associated with gastrointestinal illness [2]. A subset of the presentations accompanied by COVID-19 on the primary phase is intestinal consequences, like vomiting and diarrhea [2]. Diagnosing gastrointestinal symptoms may forerun respiratory manifestations during COVID-19 that is essential for understanding early management [1].

COVID-19 pneumonia is commonly managed with oxygenation, anti-infective drugs, anticoagulants, antiplatelet, steroids, and intravenous fluids $[3,4]$.

\section{CASE PRESENTATION}

A 70-year-old housewife, Egyptian female patient presented to the emergency department (ED) with fever, diarrhea, and vomiting. Fatigue and anorexia were initial associated symptoms. Fever, diarrhea, and vomiting continued for 7 days. The patient has a recent history of contact with her daughter for a few days. Her daughter was a confirmed COVID-19 and died within 5 
days of bilateral pneumonia in the isolated hospital. Upon general physical examination; generally, the patient was pale, sweaty, irritable, with a regular tachycardia of VR $100 \mathrm{bpm}$, blood pressure of $110 / 80 \mathrm{mmHg}$, respiratory rate of $20 \mathrm{bpm}$, the temperature of $39.7^{\circ} \mathrm{C}$, and pulse oximeter of oxygen $(\mathrm{O} 2)$ saturation of 97 $\%$. There was mild generalized superficial abdominal pain. No local tenderness, rebound, rigidity, specific abdominal lesion. No more relevant clinical data were noted during the clinical examination. She was admitted to the isolation department in the central hospital with COVID-19 acute gastroenteritis. Initially, the patient was treated with Ringer solution $(500 \mathrm{ml}$ over 30 minutes twice daily). Cold compresses were used. The patient was maintain treated with SC enoxaparin $20 \mathrm{mg}$ twice daily), aspirin tablet (75 mg, once daily), clopidogrel tablet (75 $\mathrm{mg}$, once daily), cefotaxime; (1000 mg IV every 8hours), azithromycin (500 mg PO single daily dose on the day one, followed by $250 \mathrm{mg}$ PO daily on for 5 days), oseltamivir (75 mg PO twice daily only for 5 days), hydrocortisone sod succinate (100 mg IV every 8 hours), and paracetamol $(500 \mathrm{mg}$ IV every 8 hours as needed). The patient was monitored for temperature and blood pressure. The initial complete blood count (CBC); $\mathrm{Hb}$ was $8.8 \mathrm{~g} / \mathrm{dl}$,
RBCs; $3.53 * 10^{3} / \mathrm{mm}^{3}$, WBCs; $8.9 * 10^{3} / \mathrm{mm}^{3}$ (Neutrophils; $88.4 \%$, Lymphocytes: $8.3 \%$, Monocytes; 3.3), Platelets; $125^{*} 10^{3} / \mathrm{mm}^{3}$. Measured random blood sugar was; $181 \mathrm{mg} / \mathrm{dl}$. S. Ferritin was normal; $28 \mathrm{ng} / \mathrm{ml}$. D-dimer was high $(6.64 \mathrm{mg} / \mathrm{L})$. The initial CRP was high; 87 $\mathrm{g} / \mathrm{dl}$. SGPT was high; 59U/L, SGOT was high; $49 \mathrm{U} / \mathrm{L}, \quad \mathrm{LDH}$ was high; $775 \mathrm{U} / \mathrm{L}, \quad \mathrm{S}$. albumen;4.1 $\mathrm{g} / \mathrm{dl}$, total bilirubin; $0.6 \mathrm{mg} / \mathrm{dl}$. Serum creatinine; $1.3 \mathrm{mg} / \mathrm{dl}$ and blood urea; 75 $\mathrm{mg} / \mathrm{dl}$. Chest CT scan showing no abnormality detected (Figure 1). On the seventh day of management, the above workup was repeated. CBC showing; $\mathrm{Hb}$ was $10.2 \mathrm{~g} / \mathrm{dl}$, RBCs; $4.32 * 10^{3} / \mathrm{mm}^{3}$, WBCs; $6.4 * 10^{3} / \mathrm{mm}^{3}$ (Neutrophils; $66.5 \%$, Lymphocytes: $27.4 \%$, Monocytes; 6.1), Platelets; $118^{*} 10^{3} / \mathrm{mm}^{3}$. Measured random blood sugar was; $107 \mathrm{mg} / \mathrm{dl}$. $\mathrm{S}$. Ferritin was normal; $49 \mathrm{ng} / \mathrm{ml}$. D-dimer was was normal (0.132 mg/L). CRP was normal; 2.4 $\mathrm{g} / \mathrm{dl}$. SGPT was normal; 41U/L, SGOT was normal; $38 \mathrm{U} / \mathrm{L}, \mathrm{LDH}$ was normal; $398 \mathrm{U} / \mathrm{L}, \mathrm{S}$. albumen; $3.6 \mathrm{~g} / \mathrm{dl}$, total bilirubin was normal; $0.9 \mathrm{mg} / \mathrm{dl}$. Serum creatinine was normal; 1.1 $\mathrm{mg} / \mathrm{dl}$ and blood urea was normal; $23 \mathrm{mg} / \mathrm{dl}$. Within 7 days of the above management, the patient finally showed complete clinical and laboratory improvement.

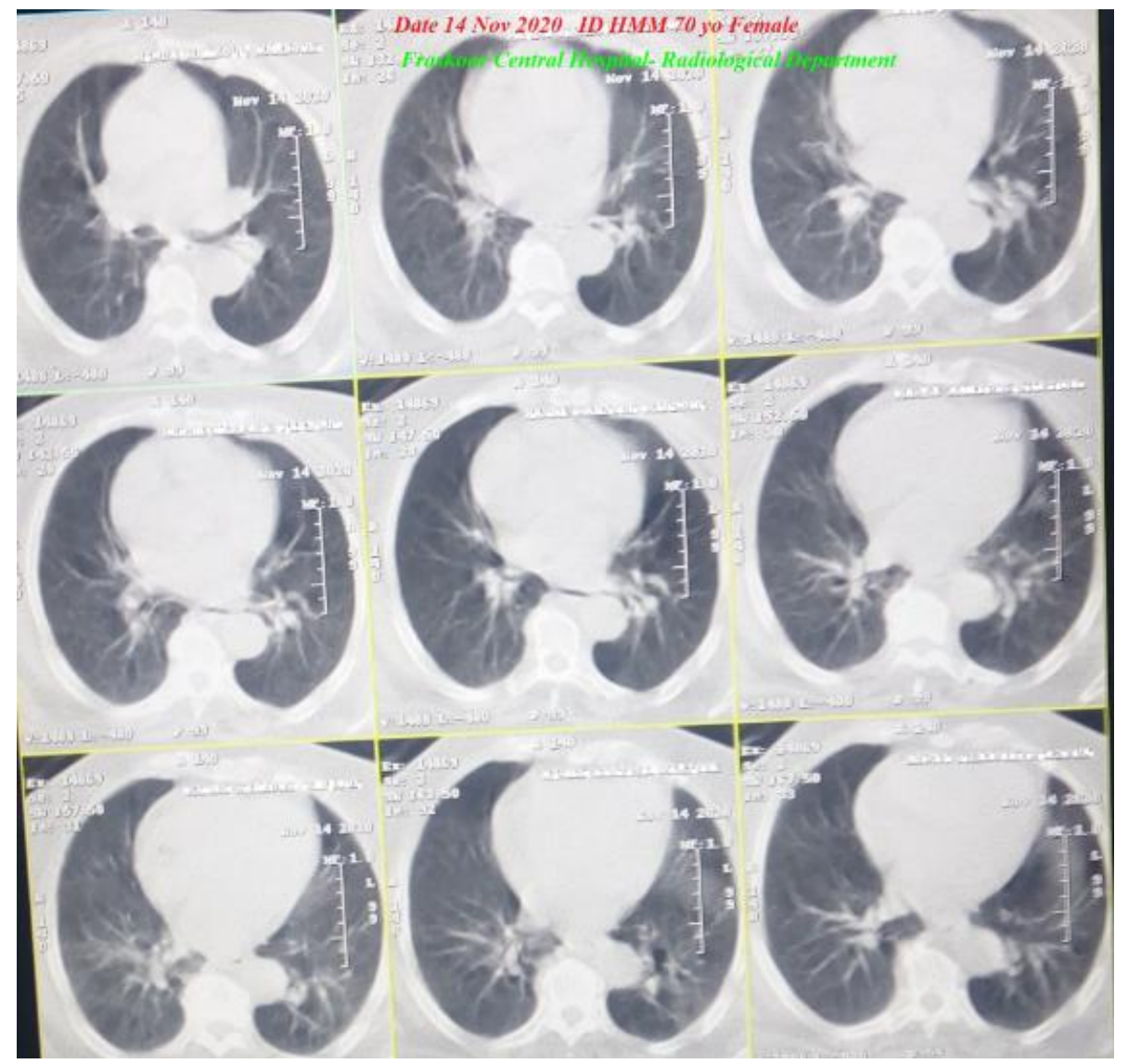

Figure1. Chest CT scan was done three days post-presentation showing no abnormality detected. 


\section{Discussion}

\section{- Overview:}

- An elderly female COVID-19 patient presented to the emergency department with acute gastroenteritis.

- The objective primary for my case study was the presence of acute gastroenteritis in a COVID-19 patient.

- The secondary objective for my case study was the question of; How did you manage the case?

- There was a history of direct contact to confirmed the COVID-19 case.

- The presence of direct contact to confirmed the COVID-19 case and lymphocytopenia on top of fever, diarrhea, and vomiting will be supporting the COVID-19 diagnosis.

- Despite the elevated d-dimer is a non-specific test but it is highly suggestive of possible associated thromboembolism.

- The presence of hypotension with tachycardia on the case presentation may be interpreted by the presence of hypovolemic shock (due to gastroenteritis).

- The blood pressure normalization urgently after fluid IV infusion with the maintenance of the effects of anti-infective drugs, anticoagulants, and antiplatelet had avoided the need for vasopressors.

- The mild elevations in liver enzymes indication of mild hepatic injury.

- It signifying the role of anti-infective drugs, anticoagulants, and antiplatelet role in COVID-19.

- Blood pressure, frequency of diarrhea, vomiting, and temperature are a strong guide for clinical follow-up in COVID-19 gastroenteritis patients.

- A dramatic normalization of the level of elevated CRP, lymphocytic count, liver enzymes, and elevated d-dimer may be used as a good laboratory guide for follow up in COVID19 gastroenteritis patients.

- The initial normal chest CT is reliable excluding the presence of pneumonia.

- I can't compare the current case with similar conditions. There are no similar or known cases with the same management for near comparison.

\section{- Limitations of the study:}

- There are no known limitations to the study.

\section{CONCLUSION AND RECOMMENDATIONS}

- The using the anti-infective drugs, anticoagulants, antiplatelet, and steroids, IV fluids in COVID-19 gastroenteritis patients are effective therapies.

-With the avoidance of oxygen inhalation, there are closed similarities between COVID-19 pneumonia and COVID-19 gastroenteritis management.

\section{ACKNOWLEDGMENT}

- I wish to thanks my wife to save time and improving the conditions for helping me.

\section{REFERENCES}

[1] Cao Y., Cai K., Xiong L. Coronavirus disease 2019: a new severe acute respiratory syndrome from Wuhan in China. Acta Virol. 2020;64:245-250. PMID: 32551792 DOI: 10.4149/av_2020_201

[2] Villapol S. Gastrointestinal symptoms associated with COVID-19: impact on the gut microbiome. Transl Res. 2020 Dec;226:57-69. doi: 10.1016/j.trsl.2020.08.004. Epub 2020 Aug 20. PMID: 32827705; PMCID: PMC7438210.

[3] Elsayed YMH. A COVID-19 Young Girl Patient with Developing Pneumonia and Septic Shock Passing the Death; A case report. Archives of Health Science. 2020-4(1)-112. DOI:10.31829/2641-7456/ahs2020-4(1)-112.

[4] Elsayed YMH. COVID-19 from Extensive Pneumonia and Acute Massive Pulmonary Embolism to Death: A Case Report. Op Acc J Bio Sci \& Res. 2(1)-2020:1-3 DOI: 10.46718/JBGSR.2020.01.000035

Citation: Yasser Mohammed Hassanain Elsayed, "A COVID-19 Acute Gastroenteritis with Similarity Pneumonia Management; Dramatic Response", International Journal of Research Studies in Medical and Health Sciences. 2020; 5(12): 36-38.

Copyright: () 2020 Yasser Mohammed Hassanain Elsayed, This is an open-access article distributed under the terms of the Creative Commons Attribution License, which permits unrestricted use, distribution, and reproduction in any medium, provided the original author and source are credited. 